\title{
Development and evaluation of degradable hydroxyapatite/sodium silicate composite for low-dose drug delivery systems
}

\author{
R MORSY \\ Biophysics Lab, Physics Department, Faculty of Science, Tanta University, Tanta 31527, Egypt
}

MS received 13 November 2015; accepted 24 February 2016

\begin{abstract}
This study was designed to develop innovative degradable hydroxyapatite (HAp)-based systems working as potential carriers for low-dose drugs. The HAp-based systems combine three components: HAp, sodium silicate and citric acid (HSC), which together could exhibit optimal characteristics as drug carriers. Both synthetic HAp (s-HAp) and extracted biological HAp (b-HAp) were used as sources of HAp due to their optimal biological properties and adsorption capacity. The s-HAp powder was prepared by co-precipitation method, while the b-HAp powder was extracted from bovine bone. Aqueous drug solutions of the atenolol, antihypertensive drug, were used as a model drug to investigate the drug release behaviour from HSC composites. The properties of s-HAP, b-HAp powders and HSC composite tablets were characterized by conventional methods. The results revealed that both s-HAp and b-HAp are pure powders and exhibited agglomerated microstructures with grain sizes less than $100 \mu \mathrm{m}$. The HSC composite tablets exhibited a dense structure, excellent compressive strength and excellent in-vitro release behaviour of atenolol. The results indicated that HAp powders and their composite tablets can be promised as economic raw materials and carriers for low-dose drugs.
\end{abstract}

Keywords. Hydroxyapatite; sodium silicate; composite; atenolol; low-dose drug; drug delivery.

\section{Introduction}

In the last decade, developing new drug delivery systems has become one of the focussed biomaterial fields due to the medical demands for controlling the level of drug release within therapeutic limits, especially for low-dose drugs [1]. However, developing suitable carriers for low-dose drugs has remained a major challenge because designing new drug carriers requires new biomaterials, technologies or tools [2]. Also, the drug carriers should have a uniform drug distribution, physicochemical stability and bioavailability upon administration [3]. Drug delivery systems based on hydroxyapatite $\left(\mathrm{Ca}_{10}\left(\mathrm{PO}_{4}\right)_{6}(\mathrm{OH})_{2} ; \mathrm{HAp}\right)$ have been used extensively in the last two decades due to the excellent biological properties of HAp such as biocompatibility, biodegradability, non-toxicity, non-inflammatory and high adsorption capacity $[4,5]$. Since HAp is the main inorganic component of natural bones, HAp-based drug delivery systems have been limited to orthopedic and dental surgeries as substitutes for damaged bones [6]. For oral drug delivery, limited efforts have focussed on developing porous HAp tablets for loading antihypertensive drugs such as metoprolol, and for loading anti-inflammatory drugs such as ibuprofen [7,8]. Low-dose drugs such as antihypertensive drugs need to develop carrier systems that confirm the optimal oral administration of

r.morsy@science.tanta.edu.eg these drugs [9]. The objective of this study was to design and characterize innovative HAp-based systems combining three components: HAp, sodium silicate and citric acids (HSC). Although the preparation of pure HAp powders, synthetic HAp (s-HAp) or biological HAp (b-HAp) now has become an interesting field of study, however, the pure HAp particles are the main challenge for the fabrication of dense HAp tablets due to the disability of HAp particles to self bind without adding chemical binders [10-13]. Therefore, the present study used sodium silicate, an inorganic binder, to overcome this drawback. Sodium silicate has been used for preparing bone cements whose properties are not strongly affected by $\mathrm{Na}$ and Si residues [14]. The binding process of sodium silicate takes place by forming a gelled $\mathrm{SiO}_{2}$ matrix that works as a binder between the ceramic particles [15]. Also, the low concentration solutions of sodium silicate have worked as nucleating agents for inducing the formation of HAp on the surface of biopolymers that were used for bone repair $[16,17]$. In addition, they are used as a $\mathrm{pH}$ adjuster in various cosmetics products $[18,19]$. However, the high alkalinity of sodium silicate is the major problem when it has been used for preparing the medical compositions [20]. In pharmaceutics, the alkalinity of medical compositions can be regulated by the addition of an acidifying agent or $\mathrm{pH}$ regulator [21]. The best selection of the acidifying agent is citric acid that has been widely used in solid oral dosage forms to modify their drug release behaviour [22-24]. Consequently, it is important to develop drug carriers based on HAp, sodium 
silicate and citric acid for administering low-dose drugs. Regarding the safety of using HAp, sodium silicate and citric acid for medical applications, this study hypothesized that the HAp-based systems combining these three components could provide an effective approach to design low-dose drug delivery systems. The aim of this study was to design novel HAp-based carriers for low-dose drugs. For this purpose, the pure s-HAp powder was prepared via co-precipitation route, and the b-HAp powder was extracted from bovine bones. Then, the HSC composite tablets were fabricated by using three components: s-HAp or b-HAp powder as a main component, sodium silicate solution as a binder and citric acid solution as a buffering agent. Sodium silicate was used for enhancing self-binding of HAp particles. The X-ray diffraction (XRD), Fourier transform infrared spectroscopy (FTIR), scanning electron microscopy (SEM), X-ray fluorescence spectrometer (XRF), mechanical and friability testings were achieved. In addition, in-vitro drug release testing was performed using atenolol, an antihypertensive drug, as a model low-dose drug for controlled drug delivery.

\section{Materials and methods}

\subsection{Materials}

Calcium hydroxide, orthophosphoric acid (85\%), sodium silicate and citric acid were purchased from Adwic, El-Nasr Chemical Co. Ltd. (Cairo, Egypt). Atenolol was a gift from Jedco International Pharmaceutical Co. (Cairo, Egypt). All reagents were analytical grade, and were used without further purification.

\subsection{Preparation of s-HAp by co-precipitation method}

s-HAp powder was prepared using the co-precipitation method according to the following procedures. Half a mole of calcium hydroxide was suspended in $500 \mathrm{ml}$ of distilled water, and then heated at $60^{\circ} \mathrm{C}$ with constant stirring. Five hundred milligrams of a solution containing 0.3 moles of phosphoric acid $(85.0 \%)$ was prepared. Then, aqueous $\mathrm{H}_{3} \mathrm{PO}_{4}$ was added slowly to $\mathrm{Ca}(\mathrm{OH})_{2}$ suspension until a $\mathrm{Ca} / \mathrm{P}$ molar ratio of 1.67 is reached. The resulting HAp slurry was allowed to boil for $60 \mathrm{~min}$, filtered and then dried in the oven at $80^{\circ} \mathrm{C}$ for $24 \mathrm{~h}$.

\subsection{Preparation and extraction of b-HAp powder}

b-HAp was hydrothermally derived from bovine bone according to the following procedures. The fresh bones were cleaned and crushed. Five hundred grams of bones were added to 21 of distilled water and then placed in a commercial stainless steel autoclave. The autoclave was tightly sealed and heated at $130^{\circ} \mathrm{C}$ for $60 \mathrm{~min}$ to remove the contamination and to obtain sterilized and cleaned bones. The bones thus obtained were filtered, washed with distilled water and then sintered at $800^{\circ} \mathrm{C}$ for $2 \mathrm{~h}$ in the furnace.

\subsection{Fabrication of HSC composites tablets}

Two groups of HSC composite tablets were fabricated by using s-HAp (s-HSC) and b-HAp (b-HSC) that were blended with sodium silicate and citric acid. Two grams of sodium silicate powder was dissolved in $15 \mathrm{ml}$ of distilled water at room temperature. Two grams of atenolol particles was dissolved in the sodium silicate solution with constant stirring. Then about $20 \mathrm{~g}$ of HAp particles and $10 \mathrm{ml} \mathrm{30 \%} \mathrm{citric} \mathrm{acid}$ solution were mixed with the solution at room temperature under vigorous stirring to form buffered slurry with $\mathrm{pH}$ value of about 7.5. The pasty mixture of each sample was divided into 50 tablets and then allowed to dry and harden at room temperature. The average weight of the tablet was about $500 \mathrm{mg}$.

\subsection{Characterization of the products}

s-HAp, b-HAp and HSC composites were characterized by using conventional methods. The crystalline structure of s-HAp, b-HAp and HSC composite tablets were characterized by XRD on a Philips PW 1840 diffractometer with $\mathrm{CuK} \alpha$ radiation of $1.5406 \AA$ at $40 \mathrm{kV}$ and $30 \mathrm{~mA}$. Peaks of the $\mathrm{X}$-ray patterns recorded for HAp samples were labelled by referring to the X-ray spectrum data obtained from the powder diffraction file (PDF) from Joint Committee on powder diffraction (JCPDS). The FTIR was obtained with PerkinElmer-1600 using $\mathrm{KBr}$ pellet technique for the range of $4000-400 \mathrm{~cm}^{-1}$. The morphologies and microstructures of the resultant products were examined by SEM using a JEOL 6400 electron microscope operating at $15 \mathrm{kV}$ of electron acceleration voltage. The chemical composition of the HSC composites was analysed using an XRF (PW-1510, Philips, Eindhoven, The Netherlands). The friability test was performed by TA-100 friabilator (ERWEKA, Hainburg, Germany). Twenty tablets of each s-HSC and b-HSC composites were weighed and rotated at velocity $25 \mathrm{rev} \mathrm{min}^{-1}$ for $5 \mathrm{~min}$. The compressive mechanical tests were performed on HSC composite tablets. Six HSC composite tablets with dimensions of $2.5 \mathrm{~mm}$ height $\times 8 \mathrm{~mm}$ diameter were used for compression strength tests. The tests were conducted at room temperature; using an Instron 4302 mechanical tester with $10 \mathrm{kN}$ load cells and a crosshead speed of $20 \mathrm{~mm} \mathrm{~min}^{-1}$.

\subsection{Drug release study from HSC composite tablets}

Atenolol release tests from different HSC composite tablets were performed at $37^{\circ} \mathrm{C}$ in $600 \mathrm{ml}$ of $0.1 \mathrm{~N} \mathrm{HCl}$ solution. HSC composite tablets were immersed in the dissolution medium and then stirred at a constant speed of $500 \mathrm{rpm}$. The samples were collected at different time points. Atenolol concentration in the sample release fluid was measured with UV/Vis spectrometer (Unico-2800) at a wavelength of $274 \mathrm{~nm}$. All experiments were done in six replicates and the mean values were reported. The paired $t$-test $(p<0.05)$ was done to statistically analyse the differences between the release results of s-HSC and b-HSC composite tablets. The errors shown in the graph represented the standard deviation 
of the percentage release as calculated from the six replicates. To study the drug release kinetics, Korsmeyer-Peppas model, equation (1), can be used to determine the mechanism of drug release from different HSC composite tablets due to the polymeric nature of silica gel matrix that binds the composite contents [25,26].

$$
\frac{M_{\mathrm{t}}}{M_{\infty}} K t^{n}
$$

where $M_{\mathrm{t}} / M_{\infty}$ is the fraction of drug released up to time $t$, $K$ the kinetic constant incorporating structural and geometric characteristic and $n$ the diffusional exponent characteristic for the drug transport mechanism. The value of $n$ indicates the drug release mechanism; if $0.1<n<0.5$, Fickian diffusion is indicated, while $0.5<n<1$ indicate s non-Fickian diffusion erosion mechanism.

\section{Results and discussion}

\subsection{Characterization of $s-H A p$ and extracted $b-H A p$ powders}

Figure $1 \mathrm{~b}$ and $\mathrm{c}$ shows the XRD pattern of s-HAp powder produced by co-precipitation method and extracted b-HAp sintered at $800^{\circ} \mathrm{C}$ for $2 \mathrm{~h}$, respectively. The $2 \theta$ values were set in the range of $10-60^{\circ}$ to investigate all significant peaks of HAp material. Both XRD patterns of s-HAp and b-HAp samples exhibit pure single phase structures with a good crystallinity as indicated by the numerous sharp peaks. All diffraction peaks are in good agreement with that of standard HAp structure (figure 1a), (JCPDS card no. 74-0874). The strong main peak sets at $2 \theta$ value of $31.7^{\circ}$, was for reflection (211). The principal diffraction peaks of HAp appears at $2 \theta$ values of 25.9, 28.9, 32.1, 32.9, 34.0, 39.2, 46.7 and $49.4^{\circ}$ were for the reflections (002), (210), (112), (300), (202), (221), (222), (213) and (004), respectively.

FTIR analysis was applied to investigate the main chemical groups that characterize HAp compound such as $\mathrm{PO}_{4}^{-3}$, $-\mathrm{OH}$ and $\mathrm{CO}_{3}^{-2}$. The FTIR spectra of s-HAp and extracted b-HAp are displayed in figure $2 \mathrm{a}$ and $\mathrm{b}$, respectively. For
$\mathrm{PO}_{4}^{-3}$ ions, there are four distinctive absorption bands that were presented at 572, 600 and $1045 \mathrm{~cm}^{-1}$ and belonged to s-HAp (figure 2a), whereas for b-HAp, they appeared at 565, 603 and $1035 \mathrm{~cm}^{-1}$ (figure 2b). For $\mathrm{OH}$ group, a distinctive broad band appeared at $3435 \mathrm{~cm}^{-1}$ for both s-HAp and b-HAp powders, whereas $\mathrm{CO}_{3}^{-2}$ group has broad peaks in the range from 1260 to $1000 \mathrm{~cm}^{-1}$.

The morphology and size of s-HAp particles and extracted b-HAp particles respectively, were characterized by SEM (figure $3 a$ and $b$ ). SEM analysis of the powders reveals dense s-HAp agglomerates with different sizes ranging between 10 and $100 \mu \mathrm{m}$. The agglomerated s-HAp grains were composed of ultrafine HAp particles that were agglomerated together by Vander Waal's forces [27]. The b-HAp grains exhibit a denser surface topography with sizes of about $10 \mu \mathrm{m}$ that can be attributed to calcinating and sintering processes at a temperature of $800^{\circ} \mathrm{C}$. These results of XRD, FTIR and SEM reveal successful preparation of microcrystalline s-HAp and successful extraction of pure b-HAp powder.

\subsection{Characterization of HSC composite tablets}

The HSC composites have a complete hardening after $20 \mathrm{~min}$. The dense HSC tablets showed very low friability i.e., less

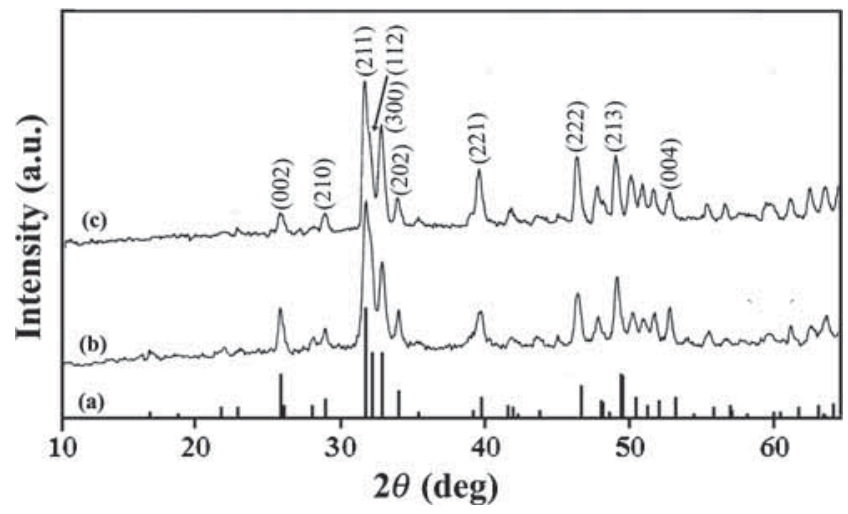

Figure 1. XRD patterns of: (a) standard HAp powder, (b) s-HAp powder and (c) b-HAp powder.

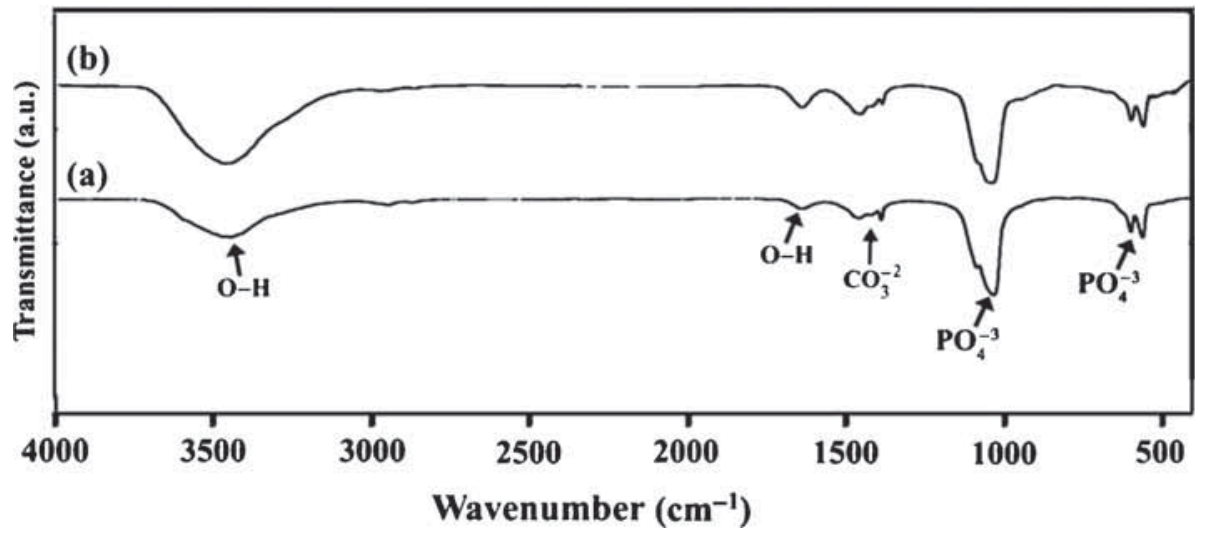

Figure 2. FTIR spectra of: (a) s-HAp powder and (b) b-HAp powder. 

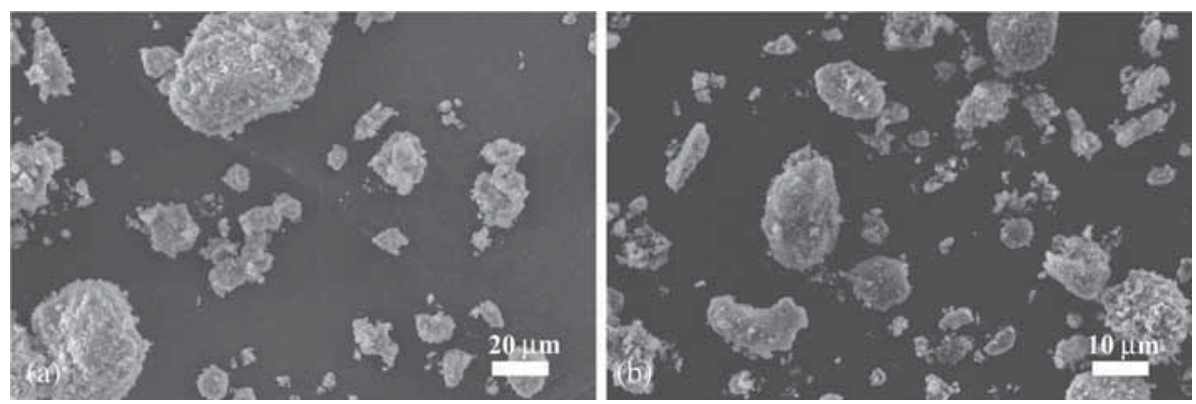

Figure 3. SEM images of: (a) s-HAp powder and (b) b-HAp powder.

Table 1. Chemical compositions of HSC composites having $2 \mathrm{~g} \mathrm{HAp}$ and $0.2 \mathrm{~g} \mathrm{Na}_{2} \mathrm{SiO}_{3}$.

\begin{tabular}{lcccc}
\hline & \multicolumn{4}{c}{ Chemical composition $(w t \% *)$} \\
\cline { 2 - 5 } HSC composite & $\mathrm{CaO}$ & $\mathrm{Na}_{2} \mathrm{O}$ & $\mathrm{SiO}_{2}$ & $\mathrm{P}_{2} \mathrm{O}_{5}$ \\
\hline s-HSC & $50.53\left(50.90^{*}\right)$ & $2.79\left(2.78^{*}\right)$ & $2.70\left(2.70^{*}\right)$ & $38.91\left(38.17^{*}\right)$ \\
b-HSC & $49.93\left(50.90^{*}\right)$ & $2.83\left(2.78^{*}\right)$ & $2.77\left(2.70^{*}\right)$ & $39.87(38.17 *)$ \\
\hline
\end{tabular}

*Theoretical values.

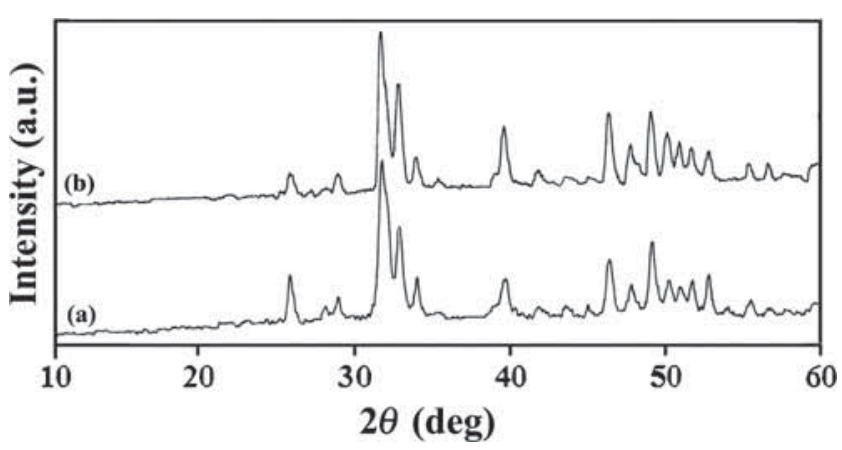

Figure 4. XRD patterns of: (a) s-HSC composite and (b) b-HSC composite.

than $0.1 \%$. The compressive strength of the dense s-HSC and b-HSC composite tablets was seen to be very high and stable within the range of $50 \pm 0.2$ and $60 \pm 0.3 \mathrm{MPa}$, respectively. The experimental chemical compositions (wt $\%$ ) of the HSC composites obtained by XRF are shown in table 1 . Comparison of the experimental values of $\mathrm{CaO}, \mathrm{Na}_{2} \mathrm{O}, \mathrm{SiO}_{2}$ and $\mathrm{P}_{2} \mathrm{O}_{5}$ content of s-HSC and b-HSC with their theoretical values has not found significant differences. The slight variations in chemical composition of b-HSC comparing with s-HSC can be ascribed to the non-stoichiometric b-HAp, which contains various natural trace elements and its $\mathrm{Ca} / \mathrm{P}$ molar ratio is not identical to 1.67 [28].

Figure $4 \mathrm{a}$ and $\mathrm{b}$ shows the XRD patterns of HSC composite tablets produced by mixing either s-HAp or extracted b-HAp powder with sodium silicate and citric acid. The addition of sodium silicate and citric acid has no effect on XRD patterns of s-HAp or b-HAp. The XRD patterns revealed the presence of a single phase structure indicating that the dominant phase in the composite is HAp phase. The reason for the

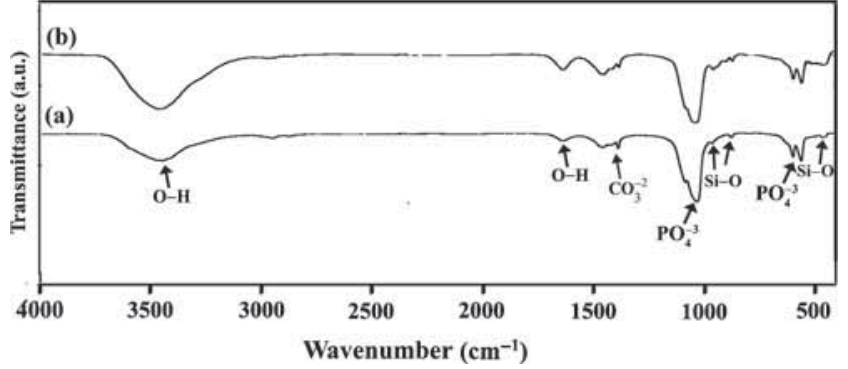

Figure 5. FTIR spectra of: (a) s-HSC composite and (b) b-HSC composite.

absence of XRD peaks belonging to sodium silicate can be attributed to its disability to crystallize due to its small weight within the composite; the weight ratio of HAp/sodium silicate was 10. It suggested that no chemical reaction occurred between the HAp and sodium silicate powder. HSC composites exhibited a strong main peak at $2 \theta$ value of $31.7^{\circ}$ and distinctive reflections identical to that of s-HAp and b-HAp (figure 1).

The FTIR spectra of HSC composites produced by mixing either s-HAp or extracted b-HAp powder with sodium silicate and citric acid are shown in figure $5 \mathrm{a}$ and b, respectively. When compared with FTIR spectra for HAp (figure 2) new absorption peaks that attributed to the vibrational modes of the $\mathrm{SiO}_{3}$ group appeared at 960,900 and $415 \mathrm{~cm}^{-1}$.

Figure 6a and b shows SEM images of HSC composite tablets produced by mixing synthetic and extracted biological HAp powders with sodium silicate and citric acid. SEM analysis of the broken tablets revealed a dense structure composed of tiny aggregated HAp particles. SEM images demonstrated that the topography of the surface after the fracture of 

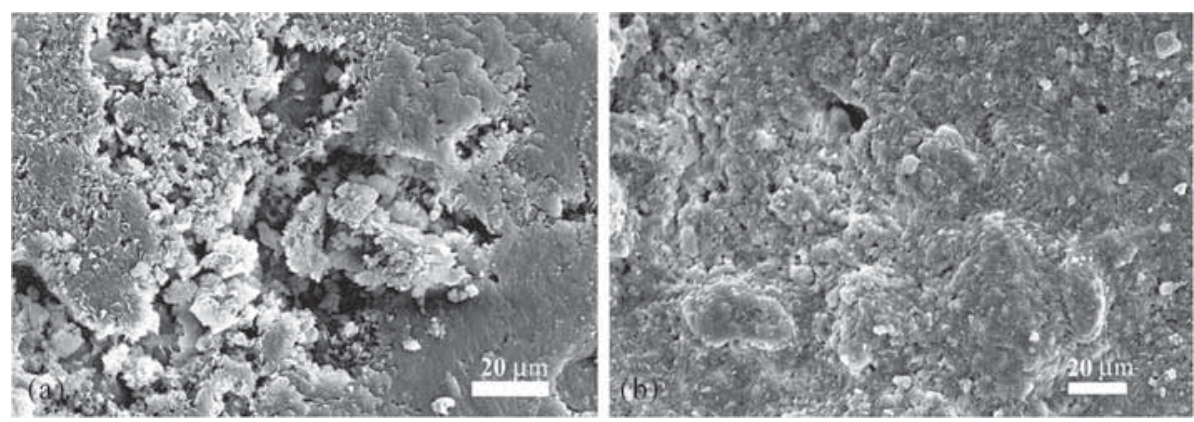

Figure 6. SEM images of: (a) s-HSC composite and (b) b-HSC composite.

the HSC composite tablet reveals minimum voids. However, the ratio of craters or cracks in the s-HSC composite tablet (figure 6a) is obvious and greater than that of the b-HSC composite tablet (figure $6 \mathrm{~b}$ ). The minimum voids are evident due to the high compatibility among HAp matrix, sodium silicate and citric acid. These results confirm the possibility to obtain s-HSC and b-HSC composite tablets, which are physically and chemically suitable as drug carriers.

\subsection{Drug release from the composite tablets}

Blending HAp powder, the main component with soluble atenolol under stirring might decrease the risk of drug inhomogeneity and particle segregation in the tablets [29,30]. HSC composite tablets were evaluated as drug carriers during the dissolution experiments due to the homogenous distribution of the aqueous drug solution inside the tablets. In general, after soaking the composite tablets in $\mathrm{HCl}$ solution, the tablets underwent a continuous degradation while their surfaces were being covered by a hydrogel matrix. The presence of the hydrogel material covering the tablets can be attributed to the behaviour of sodium silicate in aqueous media and to the dissolution of HSC matrix [19]. The behaviour of drug release from HSC composite tablets can be explained by an action of the surface erosion mechanism, where the HSC composites degrade and disappear from the surface so that their volumes decrease and shrink progressively with time. The drug delivery systems that were degraded by the mechanism of surface erosion have been preferred due to the ability to predict and control the profile of drug release [31-33]. It is observed that the supporting matrix of HSC tablets was dissolved within drug release. Figure 7 shows the cumulative atenolol release from HSC composite tablets, each contains $40 \mathrm{mg}$ of atenolol, carried out at $37^{\circ} \mathrm{C}$ in $0.1 \mathrm{~N} \mathrm{HCl}$ solution. The results show that b-HSC composite tablets have a significantly higher atenolol release rate than s-HSC composite tablets. Based on the high binding nature of HAp particles in the tablets due to sodium silicate, an immediate drug release was not anticipated. So, the burst release from these tablets was very limited. Atenolol release from b-HSC composite tablets was the fastest $(82 \%$ within $60 \mathrm{~min}$ ) when compared with s-HSC composite tablets ( $56 \%$ within $60 \mathrm{~min}$ ). This difference in the drug release behaviours from s-HSC and b-HSC composite

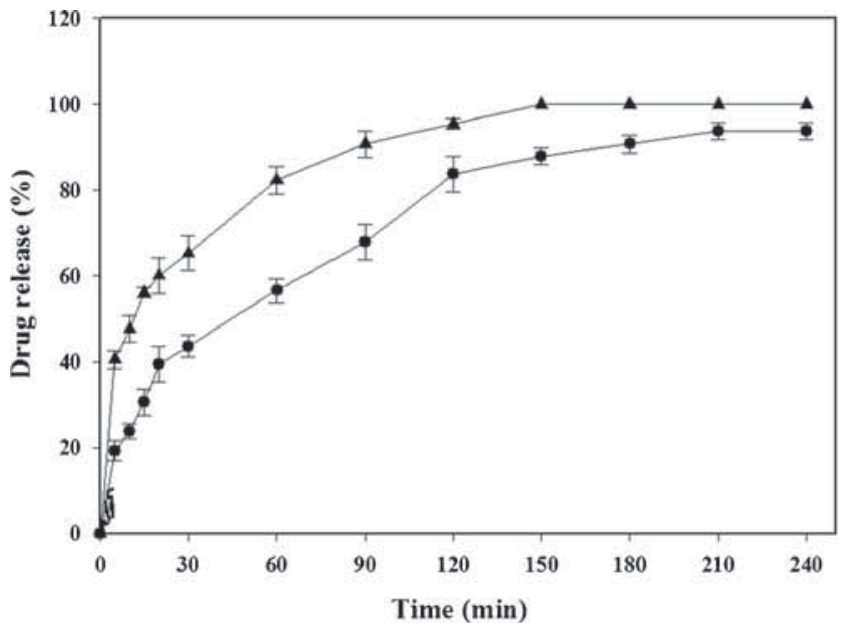

Figure 7. Drug release from HSC composite tablets containing $40 \mathrm{mg}$ atenolol, s-HSC $(\bullet)$ and b-HSC composite tablets $(\boldsymbol{\Delta})$.

tablets can be attributed to the differences between their particle sizes as shown in figure 3 . The complete release of the atenolol was achieved within $3 \mathrm{~h}$ from s-HSC (96\%) and bHSC composite tablets (100\%) indicating that interactions between the drug and tablet constitutes did not exist. After the whole degradation of HSC tablets, no residual materials have been observed. This is a clear evidence that both s-HSC and b-HSC composites allow a slower release of atenolol into $\mathrm{HCl}$ solution, whereas s-HSC composite tablets have a better drug release profile than b-HSC composite tablets.

Table 2 indicates that Peppas model gives a best-fit release kinetic data with high values of regression coefficient $\left(R^{2}\right)$. The values of $K$ were 11.06 and 30.09 for b-SHC and s-HSC composites tablets, respectively. The values of $n$ were 0.403 and 0.232 for b-SHC and s-HSC composites tablets, respectively (i.e., less than 0.5) indicating Fickian release (diffusion controlled). $R^{2}$ data indicate that Peppas model is suitably described the release of atenolol from the matrix tablets. These values indicated anomalous release mechanism with diffusion as the primary mechanism of drug release from all these composites. However, the $n$ value for s-HSC is lower than that of b-SHC, since for s-HSC, water diffusion is enhanced and the atenolol drug is dissolved and diffused out of the matrix more rapidly. The HSC composite tablets 
Table 2. In-vitro release kinetics of atenolol matrix tablets.

\begin{tabular}{lccc}
\hline & \multicolumn{3}{c}{ Korsmeyer-Peppa } \\
\cline { 2 - 4 } Composite & $\begin{array}{c}\text { Kinetic constant } \\
(K)\end{array}$ & $\begin{array}{c}\text { Release exponent } \\
(n)\end{array}$ & $R^{2}$ \\
\hline b-HSC & 11.06 & 0.403 & 0.9935 \\
s-HSC & 30.09 & 0.232 & 0.9924 \\
\hline
\end{tabular}

were found to be potentially useful in the controlled release of drugs.

\section{Conclusion}

In this study, degradable HAp-based systems for oral drug delivery application were designed as an effective alternative carrier for the controlled release of atenolol as an anti-hypertensive low-dose drug. For preparing HAp as a raw material, the s-HAp powder was synthesized by coprecipitation method, and the b-HAp powder was extracted from animal bones. The HSC composite tablets were designed by combining HAp powder with sodium silicate and citric acid. HAp was used due to its optimal biological properties and adsorption capacity, while sodium silicate was used for its ability to enhance the binding of HAp particles. The crystal structure and characteristic groups present in s-HAp, b-HAp and HSC composite tablets are evident from the XRD and FTIR, XRF data. Both s-HAp and b-HAp have microsized particles, whereas HSC composite exhibited dense surfaces. Atenolol was mixed with HSC composite and its release rate was evaluated. From these data, it can be inferred that transfer of this novel concept for low-dose tablets to industrial scale production is straightforward since the s-HAp, b-HAp and HSC composite carriers can be easily produced via direct mixing of components.

\section{References}

[1] Habraken W J E M, Wolke J G C and Jansen J A 2007 Adv. Drug Deliver. Rev. 59234

[2] Zheng J 2009 Formulation and analytical development for low-dose oral drug products (New Jersey: John Wiley \& Sons Inc.)

[3] Lakshmanan S, Gupta G K, Avci P, Chandran R, Sadasivam M, Elisa A et al 2014 Adv. Drug Deliver. Rev. 7198

[4] Zhou H and Lee J 2011 Acta Biomater. 72769

[5] Betsiou M, Bantsis G, Zoi I and Sikalidis C 2012 Ceram. Int. 382719

[6] Ginebra M P, Canal C, Espanol M, Pastorino D and Montufar E B 2012 Adv. Drug Deliver. Rev. 641090

[7] Öner M, Yetiz E, Ay V and Uysal U 2011 Ceram. Int. 372117
[8] Cosijns A, Vervaet C, Luyten J, Mullens S, Siepmann F, Van Hoorebeke L et al 2007 Eur. J. Pharm. Biopharm. 67498

[9] Alexa I F, Ignat M, Popovici R F, Timpu D and Popovici E 2012 Int. J. Pharm. 436111

[10] Sadat-Shojai M, Khorasani M T, Dinpanah-Khoshdargi E and Jamshidi A 2013 Acta Biomater. 97591

[11] Barakat N A M, Khalil K A, Sheikh F A, Omran A M, Gaihre B, Khil S M and Kim H Y 2008 Mater. Sci. Eng. C 281381

[12] Netz D J A, Sepulveda P, Pandolfelli V C, Spadaro A C C, Alencastre J B, Bentley M V L B and Marchetti J M 2001 Int. J. Pharm. 213117

[13] Son J S, Appleford M, Ong J L, Wenke J C, Kim J M, Choi S H and Oh D S 2011 J. Control. Release 153133

[14] Vernon B 2011 Injectable biomaterials: science and applications (Cambridge, UK: Woodhead Publishing Limited)

[15] Rahaman M N 2003 Ceramic processing and sintering (New York: Marcel Dekker Inc.) 2nd edn

[16] Miyaji F, Kim H-M, Handa S and Kokubo T 1999 Biomaterials 20913

[17] Oliveira A L, Malafaya P B and Reis R L 2003 Biomaterials 242575

[18] Gottschalck T E and McEwen G N 2004 International cosmetic ingredient dictionary and handbook (Washington DC: CTFA) 10th ed., Vol. 1-4

[19] Jurkić L M, Cepanec I, Pavelić S K and Pavelić K 2013 Nutr. Metab. 102

[20] Liang W, Tu Y, Zhou H, Liu C and Rüssel C 2011 J. NonCryst. Solids 357958

[21] Shoghi E, Fuguet E, Bosch E and Ràfols C 2013 Eur. J. Pharm. Sci. 48291

[22] Siepe S, Lueckel B, Kramer A, Ries A and Gurny R 2006 Int. J. Pharm. 31614

[23] Tatavarti A S and Hoag S W 2006 J. Pharm. Sci. 951459

[24] Nykänen P, Lempää S, Aaltonena M L, Jürjenson H, Veski P and Marvola M 2001 Int. J. Pharm. 229155

[25] Peppas N A 1985 Pharm. Acta Helv. 60110

[26] Korsmeyer R W, Gurny R, Doelker E, Buri P and Peppas N A 1983 Int. J. Pharm. 1525

[27] Morsy R, Elsayed M, Krause-Rehberg R, Dlubek G and Elnimr T 2010 J. Eur. Ceram. Soc. 301897

[28] Boutinguiza M, Pou J, Comesaña R, Lusquiños F, de Carlos A and León B 2012 Mater. Sci. Eng. C 32478

[29] Venables H J and Wells J I 2001 Drug Dev. Ind. Pharm. 27 599

[30] Vromans H, Poels-Janssen H G M and Egermann H 1999 Pharm. Dev. Technol. 4297

[31] Rathbone M I and Gurny R 2000 Controlled release veterinary drug delivery: biological and pharmaceutical considerations (Amsterdam: Elsevier Science)

[32] Saltzman W M 2001 Drug delivery: engineering principles for drug therapy (Oxford: Oxford University Press Inc.)

[33] Maderuelo C, Zarzuelo A and Lanao J M $2011 \mathrm{~J}$. Control. Release 1542 